\title{
ON COMPACT NORMAL SEMIGROUPS
}

\author{
by S. T. L. CHOY, B. DUMMIGAN and J. DUNCAN \\ (Received 6th December 1968)
}

\section{Introduction}

A semigroup $S$ is said to be normal if $a S=S a$ for each $a$ in $S$. Thus the class of normal semigroups includes the class of groups and the class of Abelian semigroups. Given a compact semigroup $S$ we write $P(S)$ for the convolution semigroup of probability regular Borel measures on $S$. In (3), Theorem 7, Lin asserts that a compact semigroup $S$ is normal if and only if $P(S)$ is normal. We show in this paper that Lin's result is false. In fact, if $S$ is the union of subsemigroups each of which has an identity element, we show that $P(S)$ is normal if and only if $S$ is Abelian. Thus any compact non-Abelian group contradicts Lin's result. What Lin's argument does establish is that if $P(S)$ is normal then $S$ is normal, and if $S$ is normal then $\mu P(S)=P(S) \mu$ for each point mass measure $\mu$.

In Section 1 we present some simple facts about normal semigroups. Most of the results here are probably well known but we do not know any suitable reference for them. In Section 2 we prove the result stated above about compact semigroups for which $P(S)$ is normal. We also introduce a class of semigroups, called completely normal semigroups, for which $P(S)$ is normal and give an example of a non-Abelian completely normal finite semigroup.

Lin's aim in Section 5 of (3) was to generalize some results of Glicksberg (2) to the class of compact normal semigroups. We show in Section 3 that some results can be obtained in this direction. In particular if $S$ is a compact normal semigroup we show that each idempotent measure $\mu$ in $P(S)$ is supported on a group. Thus each idempotent measure in $P(S)$ is simply the canonical extension of the Haar measure on a compact subgroup of $S$. We show also that the kernel of $P(S)$ is simply the Haar measure $m$ on the kernel of $S$, i.e. $m$ is the zero element of $P(S)$.

The first author acknowledges the financial support of an Aberdeen University Studentship.

\section{Preliminaries on normal semigroups}

Let $S$ be any semigroup and let $N$ be a subset of $S$. Then $N$ is said to be normal in $S$ if $a N=N a$ for each $a \in S$. In particular $S$ is said to be a normal semigroup if it is normal in itself. An element $c$ of $S$ is said to be central if $a c=c a$ for each $a \in S$.

Proposition 1.1. Every idempotent of a normal semigroup is central.

Proof. Let $S$ be a normal semigroup and let $j \in S$ with $j^{2}=j$. Given 
$a \in S$ there is $b \in S$ with $a j=j b$. Then $j a j=j b=a j . \quad$ A similar argument gives $j a j=j a$, so that $j$ is central.

An element $a$ of a semigroup $S$ is said to be regular if there is $b \in S$ such that $a b a=a$. In general a regular element of $S$ need not belong to a subgroup of $S$.

Proposition 1.2. Every regular element of a normal semigroup belongs to some subgroup of the semigroup.

Proof. Let $S$ be a normal semigroup and let $a, b \in S$ with $a b a=a$. Let $e=a b, f=b a$ so that $e^{2}=e, f^{2}=f$. We have $e a=a b a=a, a f=a b a=a$. Since $e, f$ are central we now have

$$
b a=b e a=e b a=e f=f e=f a b=a f b=a b=e .
$$

Thus the subsemigroup generated by $a$ and $b$ is a group with identity $e$ in which $b$ is the inverse of $a$.

Let $S$ be a normal semigroup and let $T$ be a subsemigroup of $S$. Then $T$ need not be a normal semigroup. For example in the free group on two generators $a, b$ the subsemigroup generated by $a$ and $b$ is not normal. The next result gives a sufficient condition for $T$ to be a normal semigroup.

Proposition 1.3. Let $S$ be a normal semigroup and let $T$ be a subsemigroup of $S$ such that $T$ is the union of groups. Then $T$ is a normal semigroup.

Proof. Given $a \in T$ we shall show that $T a=T \cap S a$ and similarly $a T=T \cap a S$. Since $S a=a S$ we then conclude that $T a=a T$ and $T$ is normal. It is thus sufficient to show that $T \cap S a \subset T a$ for $a \in T$. Suppose $y \in S$ and $y a \in T$. Since $T$ is the union of groups there are $e, b \in T$ with $a b=e, e a=a$. Then

$$
y a=y e a=y a b a \in T b a \subset T a
$$

and the proof is complete.

If $S$ is a compact semigroup the kernel $K$ of $S$ is the (unique) minimal closed two-sided ideal of $S$.

Proposition 1.4. If $K$ is the kernel of a compact normal semigroup $S$ then

(i) $K$ is a compact subgroup of $S$,

(ii) if $e$ is the identity of $K$ then $e j=e$ for every idempotent $j$ of $S$.

Proof. (i) Certainly $K$ is compact. Given $a \in K$ we have that $a K$ is a closed right ideal and

$$
S a K=a S K \subset a K,
$$

so that $a K$ is two-sided. Since $K$ is the kernel we have $a K=K$, and similarly $K a=K$. It is well known that $K$ is then a compact group.

(ii) Let $j \in S$ with $j^{2}=j$. Since $e$ is central $e j$ is an idempotent and it is in $K$. Thus $e j=e$.

We remark that it is easy to construct examples of compact normal semigroups that are neither groups nor Abelian. In fact let $G$ be any non-Abelian 
compact group and let $T$ be any compact Abelian semigroup that is not a group. Then the direct product semigroup $G \times T$ is such a compact normal semigroup.

\section{Compact semigroups $S$ with $P(S)$ normal}

Let $S$ be a compact semigroup and let $P(S)$ be the convolution semigroup of probability measures on $S$. For convenience of notation we shall identify the elements of $S$ with the point mass measures. Given $\mu \in P(S)$ we write supp $\mu$ for the support of $\mu$, i.e. the unique minimal closed subset $H$ of $S$ with $\mu(H)=1$. It is well known that, if $\mu, v \in P(S)$ and $0<t<1$, then

$$
\begin{aligned}
\operatorname{supp} \mu v & =\operatorname{supp} \mu \operatorname{supp} v \\
\operatorname{supp}(t \mu+(1-t) v) & =\operatorname{supp} \mu \cup \operatorname{supp} v .
\end{aligned}
$$

The theorem below shows that for a large class of compact semigroups $S, P(S)$ is normal if and only if $S$ is Abelian.

Theorem 2.1. Let $S$ be a compact semigroup with $P(S)$ normal. Let $y \in S$ with ey $=y$ for some idempotent $e$ of $S$. Then $y$ is central in $S$.

Proof. It follows from Lin's argument that $S$ is a normal semigroup and so $e$ is central in $S$. Suppose there is $x \in S$ with $x y \neq y x$. Let $\mu=t e+(1-t) x$ where $0<t<1, t \neq \frac{1}{2}$. Since $P(S)$ is normal there is $\rho \in P(S)$ with $\mu y=\rho \mu$, i.e.

$$
t y+(1-t) x y=t \rho e+(1-t) \rho x .
$$

If $H=\operatorname{supp} \rho$, then $\{y, x y\}=H e \cup H x$. If $y=x y$ then for any $w \in H$ we have $w e=y=x y=w x$, and so $y x=w e x=w x e=x y e=x y$. This contradiction shows that $y \neq x y$. We now consider various possibilities for the sets $H e$ and $H x$.

(i) $H e=\{y\}$ or $H x=\{x y\}$. It follows that there is $w \in H$ with $w e=y$, $w x=x y$. This gives

$$
y x=w e x=w x e=x y e=x y .
$$

(ii) $H e=\{x y\}, H x=\{y, x y\}$. For each $w \in H$ we have $w e=x y$ and so $w x e=w e x=x y x$. But $w x=y$ or $w x=x y$ and so $w x e=y$ or $w x e=x y$. Since both possibilities occur we obtain the contradiction $y=x y$.

(iii) $H e=\{x y\}, H x=\{y\}$. Then $\rho e=x y, \rho x=y$,

$$
t y+(1-t) x y=t x y+(1-t) y .
$$

Since $t \neq \frac{1}{2}$ this gives the contradiction $y=x y$. We are now reduced to case (iv) below.

(iv) $H e=\{y, x y\}$. Then $H x e=H e x=\{y x, x y x\}$. If $H x=\{y\}$ then $H x=H x e$ and so $y=y x=x y x$. This gives $x y=x(y x)=y=y x$. This contradiction shows that $H x \neq\{y\}$. We cannot have $H x=\{x y\}$ by part (i), and therefore $H x=\{y, x y\}=H x e$. Since $y x \neq x y$, we now deduce that $y x=y$. 
Using the normality of $P(S)$ again we get $\nu \in P(S)$ with $y \mu=\mu \nu$ and so

$$
y=t y+(1-t) y x=t e v+(1-t) x v .
$$

Let $z \in \operatorname{supp} v$ and then $e z=y=x z$. This gives $x y=x z=y=y x$. This final contradiction shows that $y$ must be central in $S$.

Corollary. If $P(S)$ is normal and $S$ is the union of subsemigroups each of which has an identity element, then $S$ is Abelian. In particular if $P(S)$ is normal and $S$ is a group then $S$ is Abelian.

Remark 1. Suppose $x, y \in S$ with $x y=y$. Then $x^{n} y=y$ for each positive integer $n$. It is well known that there is an idempotent $e$ that is a closure point of $\left\{x^{n}\right\}$ and then $e y=y$.

Remark 2. Let $S$ be any semigroup with the discrete topology and replace $P(S)$ by co $(S)$. It is then clear that Theorem 2.1 holds if compact is replaced by discrete.

Since co $(S)$ is weak* dense in $P(S)$ the result below may be established by a routine argument; we omit the proof.

Proposition 2.2. Let $S$ be a compact semigroup. Then $P(S)$ is normal if anp only if for each $x \in S, \mu \in \mathrm{co}(S)$ there are $\rho, v \in P(S)$ such that $\mu x=\rho \mu, x \mu=\mu v$.

The above result leads to a sufficient condition on $S$ that $P(S)$ be normal. Suppose that $\mu \in \operatorname{co}(S)$ so that

$$
\mu=\sum_{1}^{n} t_{r} y_{r}, t_{r} \geqq 0, \sum_{1}^{n} t_{r}=1 .
$$

Given $x \in S$ suppose there is $z \in S$ such that

$$
y_{r} x=z y_{r} \quad(r=1, \ldots, n) .
$$

The first condition of Proposition 2.2. will now be satisfied with $\rho=z$. If $S$ is normal then $E_{y}=\{z \in S: y x=z y\}$ is non-empty for each $y$ and is clearly closed. If condition (1) above holds for any finite set $\left\{y_{1}, \ldots, y_{n}\right\}$ then the closed sets $\left\{E_{y}: y \in S\right\}$ satisfy the finite intersection property. Since $S$ is compact $\cap\left\{E_{y}: y \in S\right\}$ must be non-empty. We are thus led to the following definition.

A semigroup $S$ is said to be completely normal if for each $x \in S$ there are $\phi(x), \psi(x) \in S$ such that

$$
\begin{array}{ll}
y x=\phi(x) y & (y \in S) \\
x y=y \psi(x) & (y \in S) .
\end{array}
$$

The result below is now clear.

Proposition 2.3. Let $S$ be a completely normal compact semigroup. Then $P(S)$ is normal.

We give next an example of a completely normal compact semigroup which is not Abelian. 
Example 2.4. Let $S=\{a, b, c, d, e\}$ with the following multiplication table.

\begin{tabular}{l|lllll} 
& $a$ & $b$ & $c$ & $d$ & $e$ \\
\hline$a$ & $d$ & $d$ & $e$ & $d$ & $d$ \\
$b$ & $e$ & $d$ & $d$ & $d$ & $d$ \\
$c$ & $d$ & $e$ & $d$ & $d$ & $d$ \\
$d$ & $d$ & $d$ & $d$ & $d$ & $d$ \\
$e$ & $d$ & $d$ & $d$ & $d$ & $d$
\end{tabular}

The multiplication is associative since the product of any three elements is $d$. Since $a b \neq b a, S$ is a finite non-Abelian semigroup. Define $\phi: S \rightarrow S$ by

$$
\phi(a)=c, \quad \phi(b)=a, \quad \phi(c)=b, \quad \phi(d)=d, \quad \phi(e)=e
$$

and define $\psi=\phi^{-1}$. It is readily verified that

$$
y x=\phi(x) y, x y=y \psi(x) \quad(x, y \in S)
$$

so that $S$ is completely normal.

We remark without proof that the above example is the smallest possible example of a non-Abelian completely normal semigroup. Also if $S$ is any completely normal semigroup it is easy to see that $S^{2}$ is a subset of the centre of $S$.

\section{The semigroup $P(S)$ with $S$ compact normal}

Throughout this section $S$ will be a compact normal semigroup. We write $K$ for the kernel of $S$ so that $K$ is a compact group by Proposition 1.4. We write $m$ for the Haar measure on $K$.

The results below generalize known results for compact groups and compact Abelian semigroups.

Theorem 3.1. The support of each idempotent measure in $P(S)$ is a group. Thus the idempotent measures in $P(S)$ are the Haar measures on compact subgroups of $S$.

Proof. Let $\mu \in P(S)$ with $\mu^{2}=\mu$, and let $T=\operatorname{supp} \mu$. By Collins (1), $T$ is a simple semigroup and hence is a union of subgroups. By Proposition 1.3 we have that $T$ is a normal semigroup. Since $T$ is simple we deduce that $T x=x T=T(x \in T)$. It is now well known that $T$ must be a group. Finally it is well known that the only idempotent measure supported on a compact group is the Haar measure of the group.

We write $K(P(S))$ for the kernel of $P(S)$.

Theorem 3.2. $K(P(S))=\{m\}$.

Proof. It is sufficient to show that $m$ is the zero element of $P(S)$. Given $x \in S$ we have supp $(x m) \subset K$. Since $m$ is the zero element in $P(K)$ we have $x m=x m^{2}=m$. It follows by the standard density argument that $\mu m=m$ for each $\mu$ in $P(S)$ and similarly $m \mu=m$.

E.M.S. $-\mathbf{X}$ 


\section{REFERENCES}

(1) H. S. Collins, Idempotent measures on compact semigroups, Proc. Amer. Math. Soc. 13 (1963), 442-446.

(2) I. GuicksBerg, Convolution semigroups of measures, Pacific J. Math. 9 (1959), 51-67.

(3) Y.-F. LIN, Not necessarily Abelian convolution semigroups of probability measures, Math. Zeitschrift, 91 (1966), 300-307.

DepartMent of Mathematics

King's College, AberdeEN 